\title{
Serum levels of P-selectin in men with high-functioning autism
}

Y. Iwata, K. J. Tsuchiya, S. Mikawa, K. Nakamura, Y. Takai, S. Suda, Y. Sekine, K. Suzuki,

M. Kawai, G. Sugihara, H. Matsuzaki, K. Hashimoto, M. Tsujii, T. Sugiyama, N. Takei and N. Mori

\section{Summary}

Immune dysfunction has been proposed as a mechanism for the pathophysiology of autistic-spectrum disorders. The selectin family of adhesion molecules plays a prominent role in immune/inflammatory responses. We determined the serum levels of three types of soluble-form selectin (SP, SL and $\mathrm{SE}$ ) in 15 men with high-functioning autism and 22 agematched healthy controls by enzyme-linked immunosorbent assay. Levels of SP-selectin and SL-selectin were significantly lower in patients than in controls. Furthermore, SP-selectin levels were negatively correlated with impaired social development during early childhood.

\section{Declaration of interest}

None. Funding detailed in Acknowledgements.
Immune dysfunction a potential pathophysiological mechanism of autistic-spectrum disorders. ${ }^{1,2}$ Selectins P-selectin, L-selectin and E-selectin are involved in the capture and rolling of lymphocytes along the endothelial cell surface at the first step of lymphocyte migration. ${ }^{3}$ We found abnormal serum levels of selectin in schizophrenia, ${ }^{3}$ but no studies have investigated any alterations in the serum levels of selectins in autistic-spectrum disorders We examined whether men with high-functioning autism have altered serum levels of soluble (s) forms of selectins (sP-, sLand sE-), and studied the relationship between the serum selectin levels and clinical features in these men.

\section{Method}

Fifteen men with high-functioning autism and no other comorbid neuropsychiatric disorder were recruited from the Association for Asperger Syndrome and Learning Disorders, Nagoya, Japan. All were assessed using the Japanese version of the Autism Diagnostic Interview-Revised (ADI-R). ${ }^{4}$ Results of the ADI-R and other cognitive instruments used to assess the men are given in the online supplement to this paper. All patients were unmedicated. A control group of 22 healthy volunteers was recruited from university students and community volunteers. Control participants had no developmental delay or history of psychiatric disorder or treatment. Controls were excluded if they had a firstor second-degree family history of psychiatric illness. All patients and controls were Japanese, unrelated, and free of both chronic and acute physical illnesses such as infectious or immune diseases. The mean age did not differ significantly between the two groups; 22.8 years $($ s.d. $=2.5)$ for patients and 23.2 years $($ s.d.=2.4) for controls $(P=0.64)$.

Serum samples were obtained from peripheral venous blood between 11:00 and 12:00 am after fasting for at least $3 \mathrm{~h}$, and were kept at room temperature for $30 \mathrm{~min}$. They were centrifuged, divided into portions and stored at $-80^{\circ} \mathrm{C}$. Levels of sP-, sLand sE-selectin were determined using sandwich enzyme-linked immunosorbent assay kits (R\&D Systems Inc. Minneapolis, USA). The samples of all patients were measured together in one assay with a set of control samples. Each sample was analysed and duplicated, and the mean value of the two measures was used for the analyses. A $P<0.05$ was considered statistically significant.

This study was approved by the ethics committee of the Hamamatsu University School of Medicine and all participants gave written informed consent before voluntary enrolment.

\section{Results}

The serum level of sP-selectin in the patients was significantly and substantially lower than that of controls, with a median of $71.7 \mathrm{ng} /$ $\mathrm{mL}($ mean $=67.1 \mathrm{ng} / \mathrm{ml}$, s.d.=14.4) and of $122.0 \mathrm{ng} / \mathrm{ml} \quad($ mean= $121.8 \mathrm{ng} / \mathrm{ml}$, s.d.=33.0) respectively (Mann-Whitney $U=27$, $P<0.001$ ) (online Fig. DS1(a)). The patient group also had a significantly decreased level of sL-selectin compared with the control group: $942.1 \mathrm{ng} / \mathrm{ml}$ v. $1043.9 \mathrm{ng} / \mathrm{ml}$; Mann-Whitney $U=76, P=0.005$. Regarding sE-selectin, there was no significant difference between the two groups: $45.4 \mathrm{ng} / \mathrm{ml}$ for patients $v$. $39.2 \mathrm{ng} / \mathrm{ml}$ for controls (Mann-Whitney $U=133, P=0.33$ ).

We first examined the correlations between serum sP-selectin levels and clinical variables among patients. There was a negative correlation between sP-selectin levels and ADI-R scores for domain A (Spearman's rank correlation $r=-0.589, P=0.021$ ) (Fig. DS1(b)); the lower the level of sP-selectin, the worse the social development in childhood. There were no marked or significant correlations between serum sP-selectin levels and other clinical variables. We then examined the correlations between serum sLselectin levels and clinical variables, and found no correlations.

\section{Discussion}

The serum levels of sP-selectin and sL-selectin were significantly lower in the patients than in the controls. As patients were unmedicated and free of physical illness, it is unlikely that the altered levels were ascribable to systemic inflammatory conditions or drug effects. Furthermore, there was little overlap in the distribution of the sP-selectin levels between the patient and control groups; in effect, all patients had lower values than the 25th percentile of the control group (Fig. DS1(a)).

Neither P-selectin nor L-selectin has been reported in the human brain; however, P-selectin is expressed on the endothelium of the blood-central nervous system (CNS) barrier and soluble L-selectin has been found in cerebrospinal fluid. ${ }^{3}$ Since both Pand L-selectin play important roles in the entry of circulating T-lymphocytes into the CNS, ${ }^{5}$ the decreased serum level of these two types of selectin observed in our study indicates an involvement of hypoactivity of T-lymphocytes in the pathophysiology of high-functioning autistic-spectrum disorders. It is possible that molecules not expressed in the brain may alter CNS function. Mice deprived of mature T-cells manifest spatial 
learning/memory impairments that are ameliorated by T-cell restoration. ${ }^{3}$ Furthermore, it has been reported that in children with autistic-spectrum disorders aberrant behaviour (e.g. irritability, lethargy, hyperactivity, stereotypy, inappropriate speech) improves during fever but returns when the child recovers. ${ }^{6}$ These behavioural improvements may involve activation of T-lymphocyte function.

Aberrant immune activity during brain development might also play a role in the neural basis of high-functioning autism. Diminished expression of P-selectin is associated with delayed neutrophil transmigration in neonatal rats. Therefore, decreased expression of P-selectin in individuals early in life may contribute to delayed leukocyte transmigration and increased susceptibility to infection, ${ }^{7}$ which may in turn damage neural tissues during CNS development. In fact, there is evidence that maternal viral infection in the first trimester can increase the risk of offspring developing an autistic-spectrum disorder. ${ }^{8}$

We recently found that the serum level of sL-selectin in patients with schizophrenia was significantly higher than that in controls, whereas the level of sP-selectin was not altered. ${ }^{3}$ Schizophrenia and (high-functioning) autistic-spectrum disorders are both viewed as neurodevelopmental disorders, and immune dysfunction has been proposed as a potential mechanism for the pathophysiology of both. ${ }^{1,2,9}$ The alterations in serum levels of the selectins in the two disorders suggest that selectins play a role in their pathophysiology. However, the existence of distinctive patterns of alterations (i.e. reduced (for high-functioning autism) $v$. elevated (for schizophrenia) selectin levels) indicates that the pathological mechanism may differ between the two disorders, although they share some phenotypic characteristics. The possible distinction may be supported by the different findings in brain histopathology and pharmacological sensitivity in these two disorders. Nevertheless, caution is required in interpretation based merely on one measurement of serum materials.

Low serum levels of platelet-endothelial adhesion molecule-1 (PECAM-1) and vascular cell adhesion molecule-1 (VCAM-1) occur in patients with high-functioning autistic-spectrum disorders. ${ }^{10}$ Since these adhesion molecules, together with selectins, play roles in the migration of leukocytes into inflammatory sites, ${ }^{5}$ our observation may reflect an impairment of the adhesion cascade, which in turn is responsible for dysregulation of the immune response in high-functioning autistic-spectrum disorders.

Reduced sP-selectin levels in our patient group were correlated with disturbances in social function during early childhood as assessed with ADI-R (Fig. DS1(b)). However, participants were over 18 years of age. It would be of interest to measure sP-selectin levels in very young children with high-functioning autisticspectrum disorders to determine whether such adhesion molecules serve as a serological marker.

This study has potential limitations. Active or previous exposure to common viral pathogens that may affect immunological marker expression (e.g. hepatitis and Epstein-Barr viruses) was not tested for. If exposure to these pathogens differed between patient and control groups (e.g. if the patients had been hospitalised for long periods, increasing risk of exposure), erroneous conclusions would be drawn. However, none of the patients was institutionalised, either at the time of the study or previously. Furthermore, if it were the case, elevated rather than reduced levels of selectin would be expected in the patient group. Factors such as cigarette smoking and exercise, which were not systematically assessed, may have confounded our findings. Studies have suggested that both factors may increase levels of sP-selectin. ${ }^{11,12}$ Although all patients in our study were nonsmokers, some controls may not have been. Individuals with autistic-spectrum disorders are less likely to engage in exercise than healthy people because of their impaired reciprocal social interactions, and restricted behavioural repertoire and interests. The possible bias arising from these potential confounders may lead to an overestimation of the results. However, the serum level of sP-selectin was found to correlate with impaired social development during early childhood. This implies that the current results cannot be ascribed entirely to such confounders that pertain to contemporary behaviour.

Soluble P-selectin was implicated in the pathophysiology of high-functioning autism, but studies using larger and younger samples are required to confirm our findings.

\section{Yasuhide Iwata, MD, PhD, Kenji J. Tsuchiya, MD, PhD, sumiko Mikawa, PhD, Kazuhiko Nakamura, MD, PhD, Yoshifumi Takai, MEd, Shiro Suda, MD, PhD, Yoshimoto Sekine, MD, PhD, Katsuaki Suzuki, MD, PhD, Masayoshi Kawai, MD PhD, Genichi Sugihara, MD, PhD, Hamamatsu University School of Medicine, Shizuoka; Hideo Matsuzaki, MD, PhD, Osaka-Hamamatsu Joint Research Center for Child Mental Development, Osaka University, Osaka; Kenji Hashimoto, PhD, Chiba University Center for Forensic Mental Health, Chiba; Masatsugu Tsujii, PhD, Faculty of Sociology, Chukyo University, Aichi; Toshiro Sugiyama, MD, PhD, Aichi Children's Health and Medical Center, Aichi; Nori Takei, MD, PhD, MSC, Hamamatsu University School of Medicine, Shizuoka, Japan, and Institute of Psychiatry, London, UK; Norio Mori, MD, PhD, Hamamatsu University School of Medicine, Shizuoka, Japan}

Correspondence: Professor Nori Takei, The Osaka-Hamamatsu Joint Research Center for Child Mental Development, Hamamatsu University School of Medicine, 1-20-1 Handayama, Higashi-ku, Hamamatsu, Shizuoka 431-3192, Japan. Email: ntakei@hama-med.ac.jp

First received 27 Jul 2007, final revision 10 Apr 2008, accepted 29 Apr 2008

\section{Acknowledgements}

Partial support from the Ministry of Health, Labour and Welfare, Japan. N.T. is recipient of a Grant-in-Aid for Science Research (B) (2) (14370288) and a grant for the National Center for Child Health and Development (19KOU-3) from the Ministry of Health, Labour and Welfare, Japan.

\section{References}

1 Cohly HHP, Panja A. Immunological findings in autism. Int Rev Neurobiol 2005; 71: 317-41.

2 Pardo CA, Vargas DL, Zimmerman AW. Immunity, neuroglia and neuroinflammation in autism. Int Rev Psychiatry 2005; 17: 485-95.

3 Iwata Y, Suzuki K, Nakamura K, Matsuzaki H, Sekine Y, Tsuchiya KJ, Sugihara G, Kawai M, Minabe Y, Takei N, Mori N. Increased levels of serum soluble Lselectin in unmedicated patients with schizophrenia. Schizophr Res 2007; 89: 154-60.

4 Lord C, Rutter M, Le Couteur A. Autism Diagnostic Interview-Revised: a revised version of a diagnostic interview for caregivers of individuals with possible pervasive developmental disorders. J Autism Dev Disord 1994; 24: $659-85$.

5 Engelhardt B, Ransohoff RM. The ins and outs of T-lymphocyte trafficking to the CNS: anatomical sites and molecular mechanisms. Trends Immunol 2005; 26: $485-95$

6 Curran LK, Newschaffer CJ, Lee LC, Crawford SO, Johnston MV, Zimmerman AW. Behaviors associated with fever in children with autism spectrum disorders. Pediatrics 2007; 120: e1386-92.

7 Lorant DE, Li W, Tabatabaei N, Garver MK, Albertine KH. P-selectin expression by endothelial cells is decreased in neonatal rats and human premature infants. Blood 1999; 94: 600-9.

8 Chess S. Follow-up report on autism in congenital rubella. $J$ Autism Child Schizophr 1977; 7: 69-81.

9 Nawa H, Takei N. Recent progress in animal modeling of immune inflammatory processes in schizophrenia: implication of specific cytokines. Neurosci Res 2006; 56: 2-13.

10 Tsuchiya KJ, Hashimoto K, Iwata $Y$, Tsujii M, Sekihe $Y$, Sugihara G, Matsuzaki H, Suda S, Kawai M, Nakamura K. Decreased serum levels of PECAM-1 in subjects with high-functioning autism: a negative correlation with head circumference at birth. Biol Psychiatry 2007; 62: 1056-8.

11 Ridker PM, Buring JE, Rifai N. Soluble P-selectin and the risk of future cardiovascular events. Circulation 2001; 103: 491-5.

12 Li N, Wallén $\mathrm{NH}$, Hjemdahl P. Evidence for prothrombotic effects of exercise and limited protection by aspirin. Circulation 1999; 100: 1374-9. 